\title{
Scale Up of Dextran Production from a Mutant of Pediococcus pentosaceus (SPAm) Using Optimized Medium in a Bioreactor
}

\author{
Seema Patel, Damini Kothari, Rishikesh Shukla, Debasish Das and Arun Goyal" \\ Department of Biotechnology; Indian Institute of Technology Guwahati; Guwahati, 781 039; Assam - India
}

\begin{abstract}
The mutant of Pediococcus pentosaceus (SPAm) produced earlier by UV-mutagenesis exhibiting higher dextransucrase activity as compared to wild-type was used. The generated mutant SPAm gave $12.2 \mathrm{mg} / \mathrm{ml}$, a $20 \%$ higher dextran than wild-type. Response surface methodology was carried out for further enhancement of dextran production. To enhance dextran production by the mutant SPAm, Plackett-Burman Design and a $2^{2}$ full factorial Central Composite Design was employed. After response optimization, the optimum concentration of sucrose and yeast extract was $5.115 \%(\mathrm{w} / \mathrm{v})$ and $0.635 \%(\mathrm{w} / \mathrm{v})$, respectively. The experimental values of dextran $36.0 \mathrm{mg} / \mathrm{ml}$ at flask level and $35.0 \mathrm{mg} / \mathrm{ml}$ at bioreactor level were in good agreement with the predicted value of $40.8 \mathrm{mg} / \mathrm{ml}$. The increase in dextran production by the mutant SPAm using the optimized medium was 3 fold higher as compared to unoptimized medium.
\end{abstract}

Key words: Pediococcus pentosaceus, dextran, Plackett-Burman Design, Central Composite Design, Response Surface Methodology

\section{INTRODUCTION}

Dextrans $\quad\left(\mathrm{C}_{6} \mathrm{H}_{10} \mathrm{O}_{5}\right) \mathrm{n}$ are a class of homopolysaccharides produced by the lactic acid bacteria belonging to the genera Lactobacillus, Leuconostoc, Streptococcus and Weissella (Katina et al., 2009; Patel et al., 2011). Dextrans consist of D-glucose units polymerized predominantly in $\alpha$ $(1 \rightarrow 6)$ linkage (Padmanabhan et al., 2003) and $\alpha$ $(1 \rightarrow 2), \quad \alpha-(1 \rightarrow 3), \quad \alpha-(1 \rightarrow 4) \quad$ glycosidic linked branches (Kim et al., 2003). Dextrans have enormous industrial applications due to their nonionic, inert, stable, porous, gelling and pseudoplastic attributes (Patel et al., 2011). These are used as food syrup stabilizers, matrix of chromatography columns, blood plasma substitutes, antithrombogenic agents, treatment for iron deficiency anaemia, drug carriers (Purama and Goyal, 2005; Patel et al., 2011). Dextrans have also been reported to arrest the replication of human immunodeficiency virus (HIV-1), the causative agent of the dreaded AIDS (Baba et al., 1990; Ko et al., 1991). Dextran hydrogels are used in various pharmaceutical and biomedical applications such as contact lenses, cell encapsulation for drug delivery, burn wound dressing and in spinal cord regeneration (Aumelas et al., 2007). Dextrans act as protective coating against oxidation for metal nanoparticles (Bautista et al., 2005) and shield against biofouling in

*Author for correspondence: arungoyl@iitg.ernet.in 
biomaterials (Sengupta et al., 2006).) Use of dextrans have ramified into paper, metal-plating processes and enhanced oil recovery (Patel et al., 2011). Dextrans from various microbial sources have been studied and characterized to evaluate their industrial potentials (Purama et al., 2009; Majumder and Goyal, 2009; Patel et al., 2010).

The concentrations of medium components are crucial parameters regulating the microbial metabolite production. The direct and traditional measurement techniques as 'one factor at a time' used for optimization of multivariable system is time consuming, labour intensive and prone to erroneous data. Statistical approaches such as Plackett-Burman Design and Central Composite Design by Response surface methodology (RSM) are commonly used methods to optimize culture medium, enzyme synthesis, aqueous two phase separation of proteins and glucan production (Liu and Wang, 2007; Majumder et al., 2009a, Majumder et al., 2009b). To enhance the production of dextran concentration, combinatorial interactions of medium components is a good strategy. Considering the multifarious utility of dextran, a natural isolate of lactic acid bacterium, Pediococcus pentosaceus (SPA) producing significantly high dextran concentration was screened from the soil of Assam. The dextran production ability of Pediococcus genus was reported for the first time (Patel and Goyal, $2010 b$ ). Further this was the first report on Pediococcus pentosaceus showing dextran production which is more or comparable to other commercial Leuconostoc strains (Majumder et al., 2009a; Majumder et al., 2009b). In the present work, a high dextran yielding mutant (SPAm) developed from wild-type Pediococcus pentosaceus by UV-mutagenesis was used. The dextran concentration from the mutant SPAm was enhanced by statistical optimization of the medium. Plackett-Burman Design was used to screen the significant factors and Central Composite Design was used to investigate their interactive effects.

\section{MATERIAL AND METHODS}

\section{Culturing of mutant (SPAm) of Pediococcus pentosaceus}

The mutant SPAm (Patel and Goyal, 2010a) was obtained from the wild-type Pediococcus pentosaceus (Patel and Goyal, 2010b). The cultures were maintained as stab in modified MRS agar (Goyal and Katiyar, 1996) (containing 2\% (w/v) sucrose) at $4^{\circ} \mathrm{C}$ and sub-cultured every 2 weeks. From the cultures maintained as MRS agar stab at $4^{\circ} \mathrm{C}, 1$ loopful was inoculated in the enzyme production medium described by Tsuchiya et al. (Tsuchiya et al., 1952) and grown at $25^{\circ} \mathrm{C}$ at $180 \mathrm{rpm}$ for $12 \mathrm{~h}$. This medium consisted of (\%, w/v) sucrose, 2; yeast extract, 2; $\mathrm{K}_{2} \mathrm{HPO}_{4}, 2$; $\mathrm{MgSO}_{4} . \quad 7 \mathrm{H}_{2} \mathrm{O}, \quad 0.02 ; \quad \mathrm{MnSO}_{4} .4 \mathrm{H}_{2} \mathrm{O}, \quad 0.001$; FeSO4.7 $\mathrm{H}_{2} \mathrm{O}, 0.001 ; \mathrm{CaCl}_{2}, 0.001 ; \mathrm{NaCl}, 0.001$ and the $\mathrm{pH}$ of medium was adjusted to 6.9 . $1 \%$ of this broth was transferred to $250 \mathrm{ml}$ Erlenmeyer flask containing $50 \mathrm{ml}$ of statistically designed medium with variable composition and incubated at the above mentioned culture conditions.

\section{Estimation of dextran concentration}

The carbohydrate content in the cell free supernatant of the lactic acid bacterium mutant SPAm grown in statistically designed enzyme production medium with variable ingredient composition was determined by phenol-sulphuric acid method (Dubois et al., 1956) in a micro-titre plate (Fox and Robyt, 1991). To $25 \mu$ l of sample containing dextran in a microtitre plate, $25 \mu \mathrm{l}$ of $5 \%(\mathrm{v} / \mathrm{v})$ phenol was added. The mixture was mixed by shaking the plate on a vortex mixer for 30 s. Then the plate was placed on an ice bath and $125 \mu 1$ of concentrated sulphuric acid was added to each well containing the mixture. The plate was again shaken for 30 s to ensure proper mixing of the contents of the wells. Then the plate was wrapped in cling film and incubated in water bath at $80^{\circ} \mathrm{C}$ for $30 \mathrm{~min}$. After cooling to room temperature, the absorbance was determined at $490 \mathrm{~nm}$ on a multimode microplate reader (Tecan, model Infinite ${ }^{\mathrm{TM}}$ 200). A Standard graph was plotted using dextran $(40 \mathrm{kDa})$ in the concentration range $0.1-1 \mathrm{mg} / \mathrm{ml}$.

\section{Optimization procedure and experimental designs}

Statistical designs were applied in two steps. The first step was to identify the significant nutrients for dextran production using Plackett-Burman Design (Plackett and Burman, 1946) and the second step was to optimize these significant nutrients using Central Composite Design (CCD) in response surface methodology (RSM). The experimental design and statistical analysis of the 
data were done by Minitab statistical software (Majumder et al., 2009a).

\section{Plackett-Burman Design}

Five medium components were selected for Plackett-Burman Design viz. sucrose, yeast extract, $\mathrm{K}_{2} \mathrm{HPO}_{4}$, Tween 80 and $\mathrm{CaCl}_{2}$. Among the nutrients, sucrose was selected as the carbon source as it is the substrate and inducer of dextran production (Tsuchiya et al., 1952) full stop needed. Yeast extract powder was chosen as the nitrogen source for its significant effect on dextran production (Majumder et al., 2009a). $\mathrm{K}_{2} \mathrm{HPO}_{4}$ was chosen as it acts as a buffering agent in the fermentation medium to maintain its $\mathrm{pH}$ for a longer duration (Goyal and Katiyar, 1997). The surfactant Tween 80 was selected as it alters the membrane permeability and enhances the release of the extracellular dextransucrase, which is expected to increase dextran biosynthesis (Goyal and Katiyar, 1997). $\mathrm{CaCl}_{2}$ was considered for its stimulatory effect on dextransucrase production (Qader et al., 2008). Each of the 5 factors was examined in two levels: low level (-1) and high level (+1) (Plackett and Burman, 1946). The factors considered, their levels and the design matrix were presented in Table 1. Plackett-Burman experimental design is based on the first order polynomial model:

$$
Y=\beta_{o}+\sum \beta_{i} x_{i}
$$

Where, $Y$ is the response (dextran concentration), $\beta_{o}$ is the model intercept and $\beta_{i}$ is the linear coefficient, and $x_{i}$ is the level of the independent variable.

This model is useful for screening and evaluation of the key factors that influence the response. In this work, 5 variables were screened in 16 run orders. The experiments were carried out in duplicate and the averages of the dextran concentrations were taken as response (Table 1). From the regression analysis the variables, which were significant at $95 \%$ level $(\mathrm{P}<0.05)$ were considered to have greater impact on dextran production and were further optimized by a central composite design. The experimental design and statistical analysis of the data were done by Minitab statistical software package (Version15) (Majumder et al., 2009a).

Table 1 - Plackett-Burman Design for two levels of 5 variables in uncoded values along with the observed dextran concentrations.

\begin{tabular}{ccccccc}
\hline Run Order & Sucrose (A) & Yeast extract $(\mathbf{B})$ & $\mathbf{K}_{\mathbf{2}} \mathbf{H P O}_{\mathbf{4}}(\mathbf{C})$ & $\mathbf{T w e e n} \mathbf{8 0}(\mathbf{D})$ & $\mathbf{C a C l}_{\mathbf{2}}(\mathbf{E})$ & Dextran $(\mathbf{m g} / \mathbf{m l})$ \\
\hline 1 & 6 & 1 & 1 & 1 & 0.002 & 15.6 \\
2 & 1 & 1 & 3 & 0.1 & 0.0002 & 5.2 \\
3 & 6 & 1 & 1 & 0.1 & 0.0002 & 16.5 \\
4 & 1 & 3 & 1 & 0.1 & 0.0002 & 2.9 \\
5 & 1 & 3 & 1 & 1 & 0.002 & 3.0 \\
6 & 1 & 3 & 3 & 0.1 & 0.002 & 4.2 \\
7 & 6 & 3 & 1 & 1 & 0.0002 & 11.5 \\
8 & 1 & 3 & 3 & 1 & 0.0002 & 3.0 \\
9 & 6 & 1 & 3 & 1 & 0.0002 & 16.8 \\
10 & 6 & 3 & 3 & 0.1 & 0.0002 & 13.7 \\
11 & 1 & 1 & 3 & 1 & 0.002 & 5.4 \\
12 & 1 & 1 & 1 & 0.1 & 0.002 & 5.6 \\
13 & 6 & 1 & 3 & 0.1 & 0.002 & 21.7 \\
14 & 6 & 3 & 3 & 1 & 0.002 & 12.2 \\
15 & 6 & 3 & 1 & 0.1 & 0.002 & 11.8 \\
16 & 1 & 1 & 1 & 1 & 0.0002 & 7.2 \\
\hline
\end{tabular}

\section{Central Composite Design}

The $2^{2}$ full-factorial central composite design (CCD) with two medium constituents, i.e. sucrose and yeast extract was generated by Minitab statistical software. In this study, the experimental plan consisted of 13 run orders. The experiments were carried out in duplicate and the averages of the dextran concentration were taken as the response. The relationships among the variables were determined by fitting the following secondorder polynomial equation to the data obtained from 13 experiments.

$Y=\beta_{o}+\sum_{i=1}^{k} \beta_{i} X_{i}+\sum_{i=1}^{k} \beta_{i i} X_{i}^{2}+\sum_{i} \sum_{j} \beta_{i j} X_{i} X_{j}$ 
Where, $Y$ is the predicted response, $k$ is the number of factor variables, $\beta_{o}$ is the model constant, $\beta_{i}$ is the linear coefficient, $\beta_{i i}$ is the quadratic coefficient, $\beta i j$ is the interaction coefficient. $X_{i}$ is the factor variable in its coded form. The data were analyzed statistically by ANOVA method. Pvalues below 0.1 were regarded as statistically significant. The quality of the fit of the polynomial model equation was expressed by the coefficient of determination $R^{2}$ and the significance of the regression coefficients were checked by F-test and p-value (Zhong and Wang, 2010).

\section{Validation of the model at shake flask and bioreactor level}

The confirmation and validation of data under shake flask and bioreactor level was done by using the RSM optimized medium composition. In one set, the experiment was performed in $500 \mathrm{ml}$ flask with $200 \mathrm{ml}$ optimized culture medium and grown at $25^{\circ} \mathrm{C}$ at $180 \mathrm{rpm}$. The parameters like dextran concentration, enzyme activity, $\mathrm{pH}$, sucrose concentration and cell optical density were analyzed at every $2 \mathrm{~h}$ interval as described above. In another set of experiment the fermentation was carried out in $1 \mathrm{~L}$ volume of culture medium in a $3 \mathrm{~L}$ bioreactor (Applikon, model Bio Console ADI 1025). $1 \%$ inoculum from $12 \mathrm{~h}$ grown culture was inoculated in the bioreactor. The $\mathrm{pH} 7.0$ and temperature $25^{\circ} \mathrm{C}$ were kept constant throughout the fermentation process. The Dissolved Oxygen (DO) was adjusted to $100 \%$ before inoculation. The agitation was set to $180 \mathrm{rpm}$ at the beginning of the run but changed accordingly to keep the DO above $40 \%$. The parameters like dextran concentration, enzyme activity, sucrose concentration and cell optical density were analyzed at every $2 \mathrm{~h}$ interval. The cell optical density was taken at $600 \mathrm{~nm}$. The dextran concentration was measured as described in earlier section. The enzyme activity was analysed by estimating the reducing sugars by the NelsonSomogyi protocol (Nelson, 1944; Somogyi, 1945). The sucrose concentration was determined by estimating the reducing sugars by the method of Sumner and Sisler (1944).

\section{RESULTS AND DISCUSSION}

\section{Estimation of dextran of wild-type Pedio-coccus pentosaceus and its mutant SPAm}

The mutant SPAm showed $12.2 \mathrm{mg} / \mathrm{ml}$ dextran, whereas the wild-type Pediococcus pentosaceus exhibited $10.2 \mathrm{mg} / \mathrm{ml}$. The mutant showed higher dextran production by $20 \%$ as compared to the wild-type. The dextran structure from the mutant as analysed by FTIR, NMR spectroscopy was identical to that of wild-type, however, surface morphology by SEM analysis showed larger pore size of dextran from the mutant (data not shown).

\section{Fitting the model}

The interpretation of data in Table 1 revealed that there was an extreme variation in the dextran concentration in the 16 trials ranging from 2.9 $\mathrm{mg} / \mathrm{ml}$ to $21.7 \mathrm{mg} / \mathrm{ml}$. This variation can be attributed to the variable medium composition. Regression coefficients of the 5 ingredients were analysed (Table 2).

Table 2 - Statistical analysis of Plackett-Burman Design showing coefficient, $\mathrm{T}$ and $\mathrm{P}$ values for each variable

\begin{tabular}{lccc}
\hline Variable & Coefficient & T-value & P-value \\
\hline Intercept & 9.762 & 22.81 & 0.000 \\
Sucrose (A) & 5.212 & 12.16 & 0.000 \\
Yeast extract(B) & -1.987 & -4.63 & 0.001 \\
$\mathrm{~K}_{2} \mathrm{HPO}_{4}(\mathrm{C})$ & 0.500 & 1.18 & 0.264 \\
$\mathrm{Tween}_{\mathrm{C}}(\mathrm{D})$ & -0.438 & -1.01 & 0.338 \\
$\mathrm{CaCl}_{2}(\mathrm{E})$ & 0.175 & 0.39 & 0.702 \\
\hline
\end{tabular}

Lower the P-value higher the significance of the variable (Majumder et al., 2009a). The variable sucrose and yeast extract had confidence levels greater than $95 \%$ as revealed by Pareto chart, so were considered significant. Sucrose having coefficient 5.212 was positively significant and its presence in the medium in higher concentrations enhanced dextran production. Yeast extract having coefficient -1.987 was found negatively significant and its presence in the medium in lower concentrations stimulated dextran production. Excluding insignificant variables the model equation for dextran concentration after screening by Plackett-Burman Design can be written as 
$Y=9.762+5.212 \mathrm{~A}-1.987 \mathrm{~B}$

Where, $Y=$ Dextran concentration, $\mathrm{A}=$ Sucrose and $\mathrm{B}=$ Yeast extract

On the basis of the significant coefficients (Table 2 ), sucrose and yeast extract were selected for further medium optimization to maximize dextran production. All other variables used in all the trials were kept to their median levels. At the end of the screening experiments for different nutritional factors, the conditions were optimized by Central Composite Design (CCD). Taking five levels of both the significant factors, thirteen experiments were carried out from the design (Table 3). The results of the second order response surface model fitting in the form of ANOVA are given in Table 4. To test the fit of the model, the regression equation and determination coefficient $R^{2}$ were evaluated. The model presented a high $R^{2}$ value of $94.35 \%$ and adjusted $R^{2}$ value of $90.32 \%$ for dextran concentration. The coefficients of regression for the dextran concentration were calculated and the following regression equation was obtained.

$$
Y=27.2171+24.5989 X_{1}-25.3429 X_{2}-12.5250
$$$$
X_{1} X_{2}+7.5709 X_{1}^{2}-0.9282 X_{2}^{2}
$$

Where, $Y=$ Response (Dextran concentration), $X_{1}=$ Sucrose and $X_{2}=$ Yeast extract.

The ANOVA of quadratic regression models for dextran concentration demonstrated that the model is highly significant, and is evident from the Fisher's F-test with a very low probability value $[($ Pmodel $>F=0.0000)]$. The significance of each coefficient was determined by $\mathrm{t}$-values and $\mathrm{P}$ values which are listed in Table 5. Larger magnitude of t-test and smaller P-value indicates the high significance of the corresponding coefficient (Tanyildizi et al., 2005).

The result showed that the interaction, $X_{1} X_{2}$ (sucrose. yeast extract) is highly significant with a $P$ value of 0.066 and a negative coefficient of -12.5250 .

Table $3-2^{2}$ full factorial Central Composite Design matrix of two variables in uncoded units and experimental response.

\begin{tabular}{cccc}
\hline Run order & Sucrose $(\boldsymbol{\%}, \mathbf{w} / \mathbf{v}) \boldsymbol{X}_{\boldsymbol{I}}$ & Yeast extract $(\boldsymbol{\%}, \mathbf{w} / \mathbf{v}) \boldsymbol{X}_{\mathbf{2}}$ & Dextran concentration $(\mathbf{m g} / \mathbf{m l})$ \\
\hline 1 & 3.0 & 2.75 & 15.9 \\
2 & 3.0 & 2.75 & 15.8 \\
3 & 1.5 & 1.25 & 8.6 \\
4 & 4.5 & 4.25 & 23.8 \\
5 & 3.0 & 4.865 & 11.3 \\
6 & 0.885 & 2.75 & 6.8 \\
7 & 5.115 & 2.75 & 28.3 \\
8 & 3.0 & 2.75 & 15.8 \\
9 & 3.0 & 0.635 & 16.2 \\
10 & 4.5 & 1.25 & 35.7 \\
11 & 1.5 & 4.25 & 15.9 \\
12 & 3.0 & 2.75 & 15.8 \\
13 & 3.0 & 2.75 & \\
\hline
\end{tabular}

Table 4 - ANOVA of Central Composite Design for dextran concentration.

\begin{tabular}{lccccc}
\hline Source & DF & Sum of squares & Mean Square & F-value & Prob. P > F \\
\hline Model & 5 & 773.81 & 65.743 & 23.4 & 0.00 \\
Residual (error) & 7 & 46.30 & 1.138 & - & - \\
Lack of fit & 3 & 46.29 & 2.277 & 5143.5 & 0.00 \\
Pure error & 4 & 0.012 & 0.000 & 4.74 & - \\
Total & 12 & - & - & - & - \\
\hline
\end{tabular}


Table 5 - Model coefficient of dextran estimated by multiple linear regression.

\begin{tabular}{ccccc}
\hline Model Term & Coefficient & Standard error of Coefficient & T-value & P-value \\
\hline Inter-cept & 27.2171 & 15.936 & 1.708 & 0.131 \\
$X_{1}$ & 24.5989 & 14.587 & 1.686 & 0.136 \\
$X_{2}$ & -25.3429 & 14.105 & -1.797 & 0.115 \\
$X_{1}^{2}$ & 7.5709 & 4.362 & 1.736 & 0.126 \\
$X_{2}{ }^{2}$ & -0.9282 & 4.362 & -0.213 & 0.838 \\
$X_{1} X_{2}$ & -12.5250 & 5.752 & -2.177 & 0.066 \\
\hline
\end{tabular}

\section{Analysis of response surface}

The 3D response surface and 2D contour plots are the graphical representations of regression equations (Zhong and Wang, 2010). They conveniently illustrate the relationship between responses and experimental levels of each variable and the type of interactions between two test variables. The shapes of the contour plots indicate the significance of mutual interactions between the variables. Circular contour plot symbolises negligible interactions between the corresponding variables, whereas elliptical contour plot indicates the significant interactions between the corresponding variables (Zhong and Wang, 2010). The contour and surface plot representing the regression equation for yield of dextran from the mutant SPAm was presented in Figure 1.

The plot illustrated that the interaction between the independent variable sucrose and yeast extract $\left(X_{1} X_{2}\right)$ is negative but very strong. The dextran production was increased at high sucrose and low yeast extract concentration (Fig. 1).

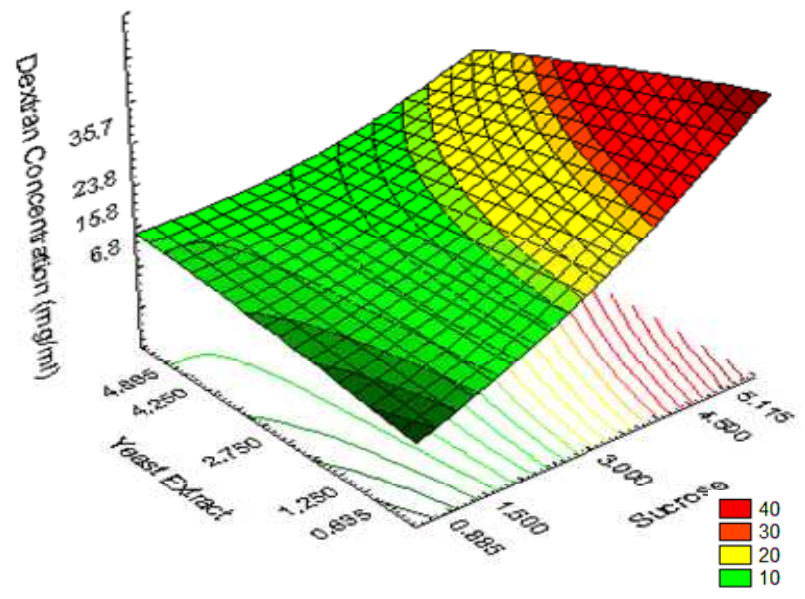

Figure 1 - Contour and surface plot of the combined effects of sucrose and yeast extract on dextran concentration of mutant (SPAm).

Optimization of medium composition and experimental validation of the model

Response optimization predicted the maximum dextran production of $40.8 \mathrm{mg} / \mathrm{ml}$ with desirability 1 , at sucrose concentration $51.15 \mathrm{~g} / \mathrm{L}$ and yeast extract concentration $6.35 \mathrm{~g} / \mathrm{L}$. Response surface methodology (RSM) showed that a medium containing $(\mathrm{g} / \mathrm{L})$ sucrose, 51.15; yeast extract, 6.35; $\quad \mathrm{K}_{2} \mathrm{HPO}_{4}, \quad 20 ; \quad \mathrm{MgSO}_{4} \cdot 7 \mathrm{H}_{2} \mathrm{O}, \quad 0.2$; $\mathrm{MnSO}_{4} \cdot 4 \mathrm{H}_{2} \mathrm{O}, \quad 0.01 ; \quad \mathrm{FeSO}_{4} \cdot 7 \mathrm{H}_{2} \mathrm{O}, \quad 0.01$; $\mathrm{CaCl}_{2} \cdot 2 \mathrm{H}_{2} \mathrm{O}, 0.01 ; \mathrm{NaCl} 0.01$ and Tween 80,5 $(\mathrm{ml} / \mathrm{L})$ was optimum for the production of dextran. The dextran production by the mutant of Pediococcus pentosaceus (SPAm) using optimized medium was compared at shake flask level and batch fermentation in a bioreactor. The fermentation profile of dextran production from the mutant in flask and bioreactor is shown in Figure 2 and Figure 3, respectively. The maximum dextran concentration obtained experimentally using the above medium composition was $36.0 \mathrm{mg} / \mathrm{ml}$ at shake flask level which showed good agreement with the predicted value $40.8 \mathrm{mg} / \mathrm{ml}$ (Fig.2).

The increase in dextran concentration of the mutant after medium optimization $(36.0 \mathrm{mg} / \mathrm{ml})$ was 3 fold higher as compared to unoptimized medium $(12.2 \mathrm{mg} / \mathrm{ml})$. For the bioreactor the 
online data such as dissolved oxygen, $\mathrm{pH}$, temperature and agitation were monitored and the offline data like dextran concentration, enzyme activity, sucrose concentration and cell optical density were plotted with time (Fig.3).

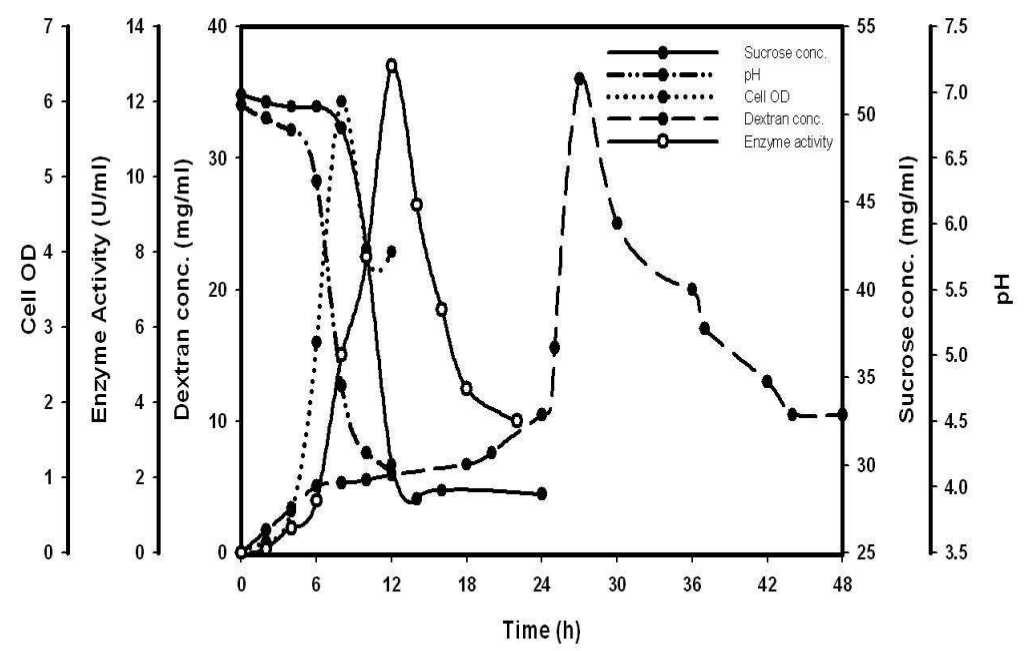

Figure 2 - Variation of dextran concentration, enzyme activity, sucrose concentration, cell optical density and $\mathrm{pH}$ during batch fermentation of the mutant of Pediococcus pentosaceus (SPAm) in flask.

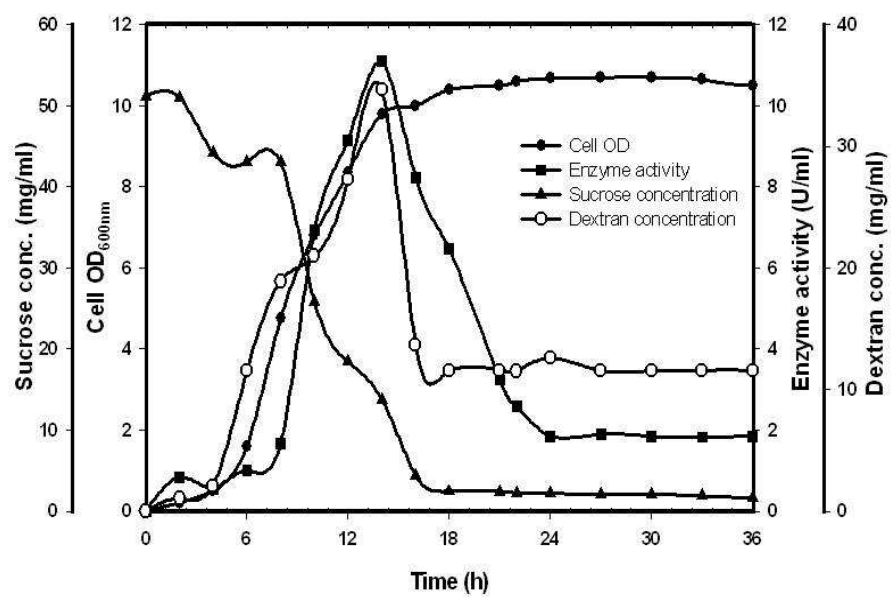

Figure 3 - Variation of dextran concentration, enzyme activity, sucrose concentration and cell optical density during batch fermentation of the mutant of Pediococcus pentosaceus (SPAm) in bioreactor.

The dextran concentration after $14-16 \mathrm{~h}$ was 35 $\mathrm{mg} / \mathrm{ml}$ which was also in good agreement with the predicted value. The $\% \mathrm{DO}$ dropped to $40 \%$ after $6 \mathrm{~h}$ of fermentation showing the micro-aerophillic nature of microorganism.

In both cases sucrose concentration profiles showed no consumption of sucrose during first 10$12 \mathrm{~h}$. On comparison of dextran and sucrose concentration profiles in the bioreactor, it was found that the dextran concentration peaked after the commencement of sucrose consumption. In bioreactor, the enzyme activity $(10.9 \mathrm{U} / \mathrm{ml})$ and the cell optical density both, reached maximum at $14 \mathrm{~h}$, which is in agreement with the report of growth associated biosynthesis of dextransucrase (Santos et al., 2000; Rodrigues et al, 2003, Michelena et al., 2003). Oxygen is known to have positive effects on the growth of certain strains of L. mesenteroides (Veljkovic et al. 1992). Thus the early production of dextran in bioreactor as 
compared to flask culture, is possibly be due to the effect of oxygen mass transfer rates on biosynthesis of dextransucrase and hence dextran.

\section{CONCLUSIONS}

Dextran concentration of Pediococcus pentosaceus (SPAm) mutant was $12.2 \mathrm{mg} / \mathrm{ml}$ with unoptimized medium. Using statistical methods the medium composition was optimized. The response optimization gave $5.115 \%$ sucrose and $0.635 \%$ yeast extract resulting in enhanced dextran production. The predicted value of dextran ( 40.8 $\mathrm{mg} / \mathrm{ml}$ ) was in good agreement with the experimental values from flask culture (36.0 $\mathrm{mg} / \mathrm{ml})$ and from bioreactor run $(35.0 \mathrm{mg} / \mathrm{ml})$. The RSM optimized medium gave 3-fold higher dextran production from the mutant SPAm as compared to unoptimized medium. The results show that the optimized medium can be implemented for large scale dextran production from Pediococcus pentosaceus (SPAm).

\section{ACKNOWLEDGEMENT}

The research work was financially supported by a project grant from Council of Scientific and Industrial Research (CSIR), New Delhi, India to AG.

\section{REFERENCES}

Aumelas, A., Serrero, A., Durand, A., Dellacherie, E., Leonard, M. (2007), Nanoparticles of hydrophobically modified dextrans as potential drug carrier systems. Colloids and Surfaces B: Biointerfaces, 59, 74-80.

Baba, M., Schols, D., De Clercq, E., Pauwels, R., Nagy, M., Gyorgyi-Edelitnyi, J., Low, M., Gorog, S. (1990), Novel sulfated polymers as highly potent and selective inhibitors of human immunodeficiency virus replication and giant cell formation. Antimicrob. Agents Chemother., 34, 134-138.

Bautista, M.C., Bomati-Miguel, O., Morales, M.P., Serna, C.J., Veintemillas-Verdaguer, S. (2005), Surface characterisation of dextran-coated iron oxide nanoparticles prepared by laser pyrolysis and coprecipitation. J. Magnetism and Magnetic Materials, 293, 20-27.
Dubois, M., Gilles, K.A., Hamilton, J. K., Rebers, P.A., Smith, F. (1956), Colorimetric method for determination of sugars and related substances. Anal. Chem., 28, 350-356.

Fox, J.D., Robyt, J.F. (1991), Miniaturization of three carbohydrate analyses using microsample plate reader. Anal. Biochem., 19, 593-596.

Goyal, A., Katiyar, S.S. (1996), Regulation of dextransucrase productivity of Leuconostoc mesenteroides $\mathrm{B}-512 \mathrm{~F}$ by the maintenance media. $J$. Gen. Appl. Microbiol., 42, 81-85.

Goyal, A., Katiyar, S.S. (1997), Effect of certain nutrients on the production of dextransucrase from Leuconostoc mesenteroides B-512F. J. Basic Microbiol., 37, 197-204.

Katina, K., Maina, N.H., Juvonen, R., Flander, L., Johansson, L., Virkki, L., Tenkanen, M., Laitila, A. (2009), In situ production and analysis of Weissella confusa dextran in wheat sourdough. Food Microbiol., 26, 734-743.

Kim, D., Robyt, J.F., Lee, S.Y., Lee, J.H., Kim, Y.M. (2003), Dextran molecular size and degree of branching as a function of sucrose concentration, $\mathrm{pH}$ and temperature of reaction of Leuconostoc mesenteroides B-512FMCM dextransucrase. Carbohydr. Res., 338, 1183-1189.

Ko, W.H., Wong, C.C., Yeung, H.W., Yung, M.H., Shaw, P.C., Tam, S.C. (1991), Increasing the plasma half-life of trichosanthin by coupling to dextran. Biochem. Pharmacol., 42, 1721-1728.

Liu, G.C., Wang, X.L. (2007), Optimization of critical medium components using response surface methodology for biomass and extracellular polysaccharide production by Agaricus blazei. Appl. Microbiol. Biotechnol., 74, 78-83.

Majumder, A, Goyal, A. (2009), Rheological and gelling properties of a novel glucan from Leuconostoc dextranicum NRRL B-1146. Food Res. Int., 42, 525-528.

Majumder, A., Singh, A., Goyal, A. (2009a), Application of response surface methodology for glucan production from Leuconostoc dextranicum and its structural characterization. Carbohydr. Polym., 75, 150-156.

Majumder, A., Bhandari, S., Purama, R.K., Patel, S., Goyal, A. (2009b), Enhanced production of a novel dextran from Leuconostoc mesenteroides NRRL B640 by response surface methodology. Ann. Microbiol., 59, 1-7.

Michelena, G.L., Martínez, A., Bell, A., Carrera, E., Valencia, R. (2003), Scale-up of dextransucrase production by Leuconostoc mesenteroides in fed batch fermentation. Braz. Arch. Biol. Technol., 46, 455-459. 
Nelson, N. (1944), A photometric adaptation of the Somogyi method for the determination of glucose. $J$. Biol. Chem., 153, 375-380.

Padmanabhan, P.A., Kim, D.S., Pak, D., Sim, S.J. (2003), Rheology and gelation of water-insoluble dextran from Leuconostoc mesenteroides NRRL B523. Carbohydr. Polym., 53, 459-468.

Patel, S., Goyal, A. (2010a), Isolation, characterization and mutagenesis of exopolysaccharide synthesizing new strains of lactic acid bacteria. Internet $J$. Microbiol., 8 (1), 1-14.

Patel, S., Goyal, A. (2010b), 16S rRNA based identification and phylogenetic analysis of a novel dextran producing Pediococcus pentosaceus isolated from north-east Indian microbial diversity. Curr. Trends Biotechnol. Pharm., 4 (3), 746-754.

Patel, S., Majumder, A., Goyal, A. (2011), Potentials of exopolysaccharides from lactic acid bacteria. Indian J. Microbiol., DOI 10.1007/s12088-011-0148-8

Patel, S., Kasoju, N., Bora, U., Goyal, A. (2010), Structural analysis and biomedical applications of dextran produced by a new isolate Pediococcus pentosaceus screened from biodiversity hotspot Assam. Bioresour. Technol., 101, 6852-6855.

Plackett, R.L., Burman, J.P. (1946), The design of optimum multifactorial experiments. Biometrika, 33, 305-325.

Purama, R.K., Goyal, A. (2005), Dextransucrase production by Leuconostoc mesenteroides. Ind. J. Microbiol., 2, 89-101.

Purama, R.K., Goswami, P., Khan, A.T., Goyal, A. (2009), Structural analysis and properties of dextran produced by Leuconostoc mesenteroides NRRL B640. Carbohydr. Polym., 76, 30-35.

Qader, S.A., Aman, A., Saeeda, B., Syed, N., Azhar, A. (2008), Role of calcium ions and temperature on dextransucrase production. Ind. J. Biotechnol., 7, 404-406.
Rodrigues, S., Lona, L.M., Franco, T.T. (2003), Effect of phosphate concentration on the production of dextransucrase by Leuconostoc mesenteroides NRRL B-512. Bioprocess. Biosyst. Eng., 26, 57-62.

Santos, M., Teixeira, J., Rodrigues, A. (2000), Production of dextransucrase, dextran and fructose from sucrose using Leuconostoc mesenteroides NRRL B-512. Biochem. Eng. J., 4, 177-188.

Sengupta, A., Wang, S., Link, E., Anderson, E.H., Hofmann, C., Lewandowski, J., Kottke-Marchant, K., Marchant, R.E. (2006), Glycocalyx-mimetic dextran modified poly (vinyl amine) surfactant coating reduces platelet adhesion on medical-grade polycarbonate surface. Biomaterials, 27, 3084-3095.

Somogyi, M. (1945), A new reagent for determination of sugars. J. Biol. Chem., 160, 61-68.

Sumner, J.B., Sisler, E.B. (1944), A simple method for blood sugar. Arch. Biochem., 4, 433-436.

Tanyildizi, M.S., Ozer, D., Elibol, M. (2005), Optimization of $\alpha$-amylase production by Bacillus sp. using response surface methodology. Process Biochem., 40, 2291-2296.

Tsuchiya, H.M., Hellman, N.N., Koepsell, H.J., Corman, J., Stringer, C.S., Rogovin, S.P., Bogard, M.O., Bryant, G., Feger, V.H., Hoffman, C.A., Senti, F.R., Jackson, R.W. (1952), Factors affecting molecular weight of enzymatically synthesized dextran. J. Am. Chem. Soc., 77, 2412-2419.

Veljkovic, V.B., Lazic, M.L., Rutic, D.J., Jovanovic, S.M., Skala, D.U. (1992), Effect of aeration on extracellular dextran production by Leuconostoc mesenteroides. Enzym. Microb. Technol., 14, 665668.

Zhong, K., Wang, Q. (2010), Optimization of ultrasonic extraction of polysaccharides from dried longan pulp using response surface methodology. Carbohydr. Polym., 80, 19-25. 


\section{PÁGINA EM BRANCO}

